\title{
Femtosecond differential transmission measurements on low temperature GaAs metal- semiconductor-metal structures
}

Keil, Ulrich Dieter Felix; Hvam, Jørn Märcher; Tautz, S.; Dankowski, S. U.; Kiesel, P.; Döhler, G. H.

Published in:

Applied Physics Letters

Link to article, DOI:

10.1063/1.119310

Publication date:

1997

Document Version

Publisher's PDF, also known as Version of record

Link back to DTU Orbit

Citation $(A P A)$ :

Keil, U. D. F., Hvam, J. M., Tautz, S., Dankowski, S. U., Kiesel, P., \& Döhler, G. H. (1997). Femtosecond differential transmission measurements on low temperature GaAs metal-semiconductor-metal structures. Applied Physics Letters, 70(1), 72-74. https://doi.org/10.1063/1.119310

\section{General rights}

Copyright and moral rights for the publications made accessible in the public portal are retained by the authors and/or other copyright owners and it is a condition of accessing publications that users recognise and abide by the legal requirements associated with these rights.

- Users may download and print one copy of any publication from the public portal for the purpose of private study or research.

- You may not further distribute the material or use it for any profit-making activity or commercial gain

- You may freely distribute the URL identifying the publication in the public portal 


\title{
Femtosecond differential transmission measurements on low temperature GaAs metal-semiconductor-metal structures
}

\author{
Ulrich D. Keil a) and Jørn M. Hvam \\ Mikroelektronik Centret, Technical University of Denmark, DK-2800 Lyngby, Denmark
}

Sönke Tautz, Stefan U. Dankowski, Peter Kiesel, and Gottfried H. Döhler

Institut für Technische Physik I, Erwin-Rommel-Str. 1, 91058 Erlangen, Germany

(Received 26 August 1996; accepted for publication 5 November 1996)

\begin{abstract}
We report on differential transmission measurements on low temperature grown (LT)-GaAs with and without applied electrical fields at different wavelengths. Electrical fields up to $100 \mathrm{kV} / \mathrm{cm}$ can be applied via an interdigitated contact structure to our LT GaAs samples which have been removed from the substrate by epitaxial lift off. In the presence of an electric field, both, the absorption bleaching due to phase space filling and field induced absorption changes due to the Franz-Keldysh effect contribute to the transmission changes. We observe an extended carrier lifetime with applied field. The response time of a biased metal-semiconductor-metal detector, therefore, exceeds the carrier life time of the substrate material. (C) 1997 American Institute of Physics. [S0003-6951(97)02601-6]
\end{abstract}

Low temperature grown (LT) GaAs layers have been used as insulating layers ${ }^{1}$ as well as photoconductive switch substrates with subpicosecond response times. ${ }^{2,3}$ The possibility of achieving an epitaxial layer with a high breakdown field, high resistivity, and short carrier lifetime qualifies this material in a unique way for the fabrication of sensitive, fast photoconductive switches. In addition, investigations of carrier dynamics under high electric fields are possible. By separating the epitaxial layer from the substrate using an epitaxial liftoff technique, the transmission measurements can be performed below and above the band gap.

The samples were prepared as follows: A $1 \mu \mathrm{m} \mathrm{GaAs}$ layer was grown by molecular beam epitaxy at $250{ }^{\circ} \mathrm{C}$ on a 50-nm-sacrificial AlAs layer. The samples were then annealed by rapid thermal annealing for $1 \mathrm{~min}$ at $600{ }^{\circ} \mathrm{C}$. Ti/Au contacts were evaporated to form metal-semiconductormetal (MSM) structures. Finally, a $\mathrm{SiO}_{x}$ antireflection coating was deposited. The investigated sample has a finger width of $1 \mu \mathrm{m}$ and spacing of $6 \mu \mathrm{m}$ and a total area of 40 $\times 40 \mu \mathrm{m}^{2}$. This finger spacing is large compared to MSM detectors that rely on a short transit time and hence a short contact spacing. ${ }^{4,5}$ For the investigation of the carrier dynamics at high fields, the $6 \mu \mathrm{m}$ spacing does not present a limitation as fields of $200 \mathrm{kV} / \mathrm{cm}$ (without illumination) can be easily applied.

The LT GaAs layer, together with the contacts, was removed from the substrate by epitaxial lift off and placed on a glass substrate. We are able to lift off circuits of up to $5 \times 5 \mathrm{~mm}^{2}$ with the electrical contacts remaining intact. For high resistivity and high breakdown fields, the annealing temperatures, $T_{a}$, was chosen to be $600{ }^{\circ} \mathrm{C}$ (Ref. 6) although we measure a slightly shorter carrier life time for $T_{a}=525{ }^{\circ} \mathrm{C}$. For $T_{a}=600{ }^{\circ} \mathrm{C}$, the conductivity, $\sigma$, can drop below that of semi-insulating material. ${ }^{7}$ This sample preparation enables the investigation of fields up to $100 \mathrm{kV} / \mathrm{cm}$ while pumping with an average optical intensity of up to 20 $\mathrm{kW} / \mathrm{cm}^{2}(50 \mathrm{~mW} / 5 \mu \mathrm{m} \times 5 \mu \mathrm{m})$ at the same time.

The laser source is a mode locked Ti:sapphire laser with 100 - fs pulse widths. The pump beam is chopped at $4 \mathrm{kHz}$,

${ }^{a)}$ Electronic mail: ulli@mic.dtu.dk the probe light is detected with a Si photodiode and measured through a lock-in amplifier. The pump beam is chopped at $4 \mathrm{kHz}$ and the associated change in the transmitted probe light is detected with a $\mathrm{Si}$ photodiode and measured through a lock-in amplifier. Pump and probe beams are cross polarized. The linear polarization of the probe beam is kept parallel to the applied electric field.

In this letter, we present differential transmission spectra (DTS) with and without applied field, different wavelengths, and different optical intensities. For an excitation wavelength of $867 \mathrm{~nm}$, the maximum carrier densities generated in our experiments are $10^{19} \mathrm{~cm}^{-3}$ (corresponding to the normalized optical intensity, $I$, denoted in the graphs). Without an applied electric field, the spectra can be determined by band filling effects, band gap renormalization, and free carrier absorption. For continuous wave excitation on GaAs, the bandgap shift for carrier densities of $10^{19} \mathrm{~cm}^{-3}$ is up to $50 \mathrm{meV}$. $^{8}$ This shift results in an increased absorption whereas phase space filling results in bleaching or reducing of the absorption. However, on LT GaAs substrates band gap renormalization has not been observed as a major contribution to DTS so far. For unannealed LT GaAs with a high impurity concentration, induced absorption has been observed. ${ }^{9}$ For the annealed samples investigated here, the impurity concentra-

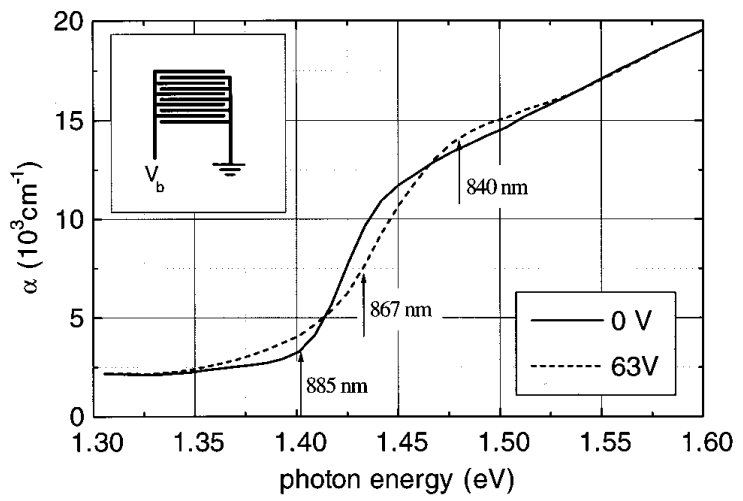

FIG. 1. Absorption spectra of the LT GaAs MSM structure with and without applied field. The applied voltage corresponds to an average field of 100 $\mathrm{kV} / \mathrm{cm}$. The indicated wavelengths are chosen for the time resolved measurements. The inset shows a sketch of the MSM layout. 


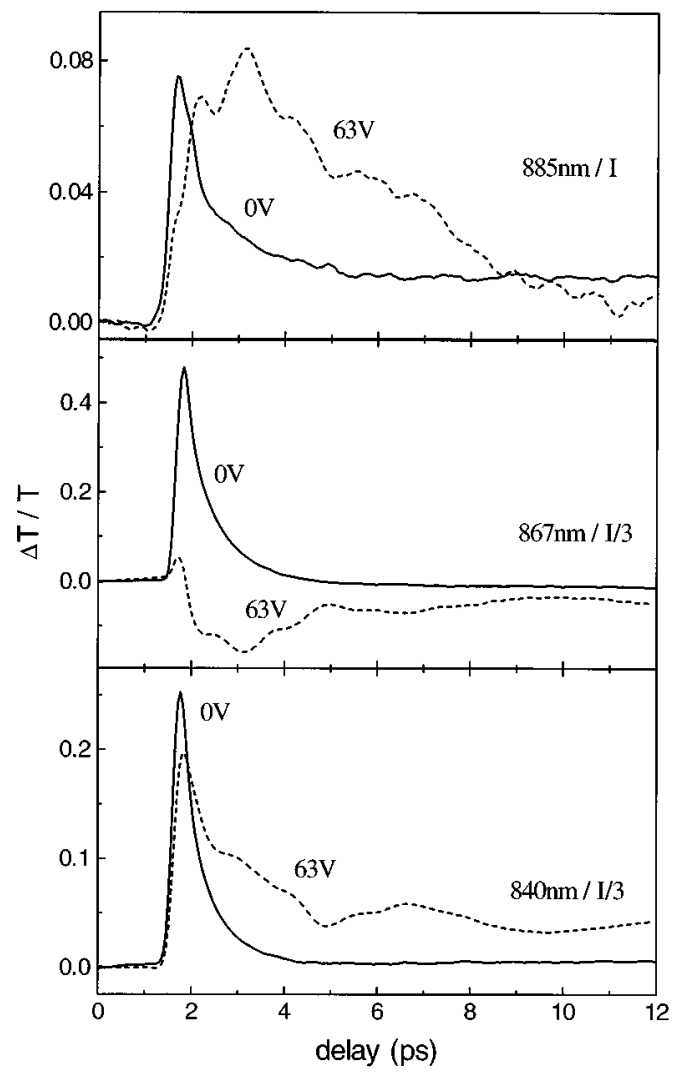

FIG. 2. DTS of the LT GaAs MSM structure with and without applied field for different wavelengths. The wavelengths and the relative optical pump intensities are indicated in the graph. At 885 and $840 \mathrm{~nm}$, field screening effects have a positive sign and at $867 \mathrm{~nm}$, the sign is negative.

tion is sufficiently small so that the DTS are expected to be determined by state filling effects. For a detailed investigation of the FK effect on LT GaAs MSM structures we refer to Ref. 10. With an applied field, we expect two main contributions to the DTS: (i) an increase of the transmission due to absorption bleaching, (ii) change of the transmission via the Franz-Keldysh (FK) effect due to screening of the applied field by drifting charge carriers. Depending on the wavelength, the latter contribution can have a positive or negative sign. Therefore, we have performed time resolved transmission measurements at three different photon energies as indicated in Fig. 1: (i) below band gap at $885 \mathrm{~nm}$ where field screening results in a decrease of the absorption coefficient, $\alpha$, (ii) directly above band gap $(867 \mathrm{~nm})$ where $\alpha$ increases, and (iii) at $840 \mathrm{~nm}$ where $\alpha$ (for this specific applied field) decreases again with field. State filling results in a saturation of the absorption at all these wavelengths. For a detailed investigation of field and wavelength dependence of the FK effect on LT GaAs MSM structures, we refer to Ref. 10 .

Figure 2 shows DTS for these three wavelengths with and without applied electric field. Without an applied field, transmission changes are positive at all wavelengths. This result shows that state filling effects dominate over possible contributions from band gap renormalization. We observe the maximum relative change in transmission, $\Delta T / T$, at 867 $\mathrm{nm}$, close to the band edge. The signal decay is expected to be dominated by recombination and shows a sub-ps decay time which is typical for LT GaAs. The decay time constant is marginally longer than at $840 \mathrm{~nm}$ where both relaxation and recombination determine the recovery time. At $885 \mathrm{~nm}$, the small residual absorption below the band gap (see Fig. 1) and the related reduced generation of charge carriers result in a factor of 5 smaller $\Delta T / T$. At this wavelength, two components can be resolved by expanding the signal scale compared to the short wavelengths: a fast, time resolution limited, component and a slowly decaying signal with a time constant longer than our time window.

The most interesting DTS are taken with an applied field (marked with $63 \mathrm{~V}$ in Fig. 2). In this case, state filling effects and FK contributions to the DTS compete. At 840 and 885 $\mathrm{nm}$, both effects contribute with the same sign, at $867 \mathrm{~nm}$ with opposite sign. This change in sign confirms that the signal after the initial peak of approximately $400 \mathrm{fs}$ is determined by field screening effects. With the applied field, the initial absorption bleaching peak is suppressed. We interpret this as follows: Due to the field induced $k$-space shift of the photogenerated carrier distribution, the bleaching effect shifts out of the observation window. In addition, we observe that in all cases the response is dramatically extended in time. The field induced drift of electrons and holes into opposite directions results in the formation of space charge regions with excess electron and hole densities. After about 2 ps, these space charges have screened the applied external field resulting in maximal FK absorption changes. The recovery time depends on an intricate interplay of electron and hole trapping, recombination, and drift. We would like to emphasize that all of these processes depend strongly on the position coordinate between the contacts. For instance, drift, trapping, and recombination processes compete with each other in the neutral region, whereas there is only trapping but no recombination in the space charge regions due to the lack of recombination partners. A systematic analysis of the time dependence of the recovery of the FK absorption is necessary to reveal detailed information about carrier drift, trapping, and recombination.

It should be pointed out that two effects contribute to a field change in our structure: (i) local field screening by the shifted electron and hole clouds, as discussed, and (ii) voltage breakdown across the whole device because the charge (and thus the energy) stored in the MSM capacitor is limited. ${ }^{11,12}$ Therefore, the dipole charge created by the spatially separating electron and hole clouds cannot exceed this stored charge for times shorter than the RC-time constant of the external circuit. In fact, this is expected to be the case in our experiments. We estimate the total number of photogenerated carriers to be about $10^{9}$, equivalent to $160 \mathrm{pCb}$. For our MSM structure, we calculate a detector capacitance of 8 $\mathrm{fF}^{5}$ and a charge of $0.5 \mathrm{pCb}$. The voltage at the electrodes cannot be assumed to be constant during the carrier acceleration process.

Figure 3 shows DTS at $63 \mathrm{~V}$ for three different optical excitation intensities. Here the transition from bleaching dominated (for high intensity) to FK dominated spectra (for low intensity) is demonstrated. Qualitatively we interpret this observation as follows: Obviously, state filling effect are stronger for high intensities as more carriers are generated. At the highest intensity, $I$, the photogenerated electron and hole density for $\lambda=867$ and $840 \mathrm{~nm}$ is of the order of 


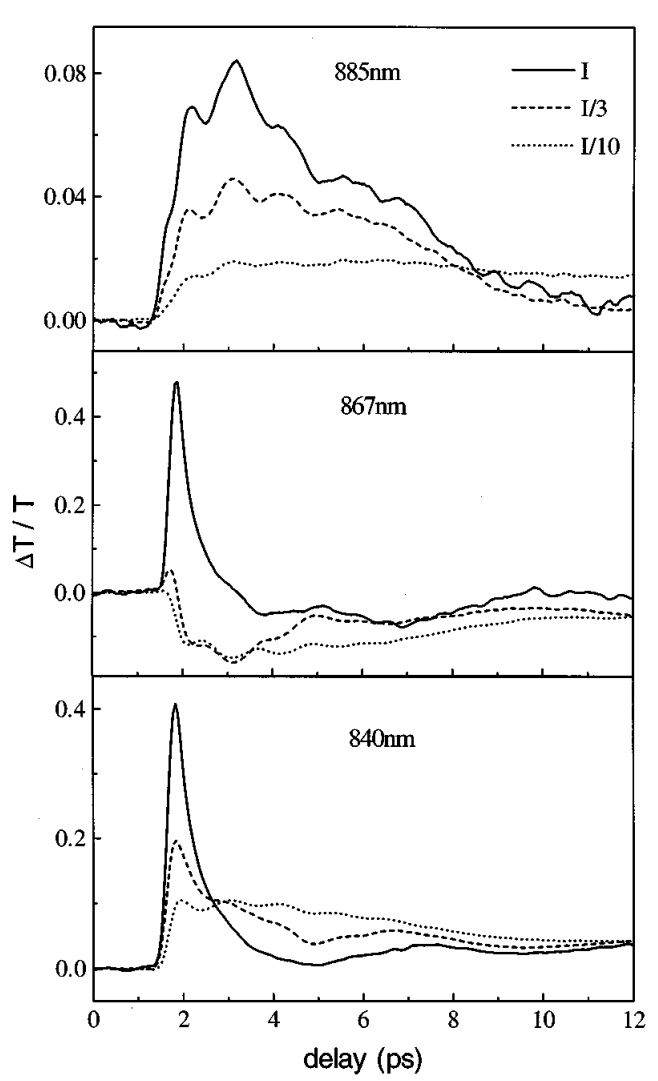

FIG. 3. DTS of the LT GaAs MSM structure with an applied field for different wavelengths and different pump intensities. State filling effects dominate for high intensities and field screening effects become more pronounced for lower intensities. The relative optical pump intensities are indicated in the graph.

$10^{19} \mathrm{~cm}^{-3}$. The time required for neutralizing the MSM capacitor charge through a small displacement of the high density electron and hole cloud in real space is so short (about $40 \mathrm{fs}$ ) that no significant displacement in momentum space occurs within this time. Hence, the bleaching by state filling can persist in spite of the applied voltage. For times longer than the carrier relaxation and recombination time, however, the signal is dominated by the slowly decaying increase (at $867 \mathrm{~nm}$ ) or decrease (at $840 \mathrm{~nm}$ ) of absorption, induced by a slow recovery of the collapsed electrical field. At reduced optical intensity, where a strong bleaching signal is still observed at zero bias, the time required for the collapse of the electrical field increases. It becomes comparable or even longer than the time necessary for shifting the carrier distribution out of the observation window in momentum space. This is clearly visible in the results for $867 \mathrm{~nm}$ at $I / 3$ displayed in Figs. 2 and 3. The fact that the bleaching signal at $\lambda=840 \mathrm{~nm}$ degrades less with decreasing intensity is consistent with the interpretation that the higher absorption results in higher electron hole densities at given $I$.

With an applied voltage, we observe additional features. The DTS exhibit oscillations of about $800 \mathrm{GHz}$ independent of applied voltage, pump intensity, and wavelength. The oscillations are most pronounced with high field and low excitation intensity. At high optical excitation, an extra oscillation of approximately $200 \mathrm{GHz}$, independent of our measurement parameters, is observed. One possibility is that we observe an "oscillating finger," assuming that part of the electrical pulse generated by the laser is reflected at the ends of the fingers of the interdigitated contacts. We assume here that the propagation speed of the guided electromagnetic wave is determined by the average refractive index of air and the substrate. For a finger length of $34 \mu \mathrm{m}$ and the electromagnetic wave propagating at $1.3 \times 10^{8} \mathrm{~m} / \mathrm{s}$, one obtains a round trip time of $500 \mathrm{fs}$ which is about a factor of two faster than the observed oscillations. This explanation is supported by our latest results which show that these oscillations are stronger for a nonuniform illumination of the MSM structure. The fact that the observed oscillation frequency and phase is independent of the wavelength, the applied field, and most of all carrier density, rules out effects related to the nonparabolicity of the GaAs band structure, i.e., Gunn-type oscillations. However, more experiments are necessary to resolve this issue.

In summary, we have measured the transient response of a thin LT GaAs MSM structure in differential transmission and resolved the carrier and field dynamics in the semiconductor. For high optical intensities and low fields, we observe very fast $(\approx 400 \mathrm{fs})$ transients determined by absorption bleaching and subpicosecond internal recombination. For low optical intensities and high fields, we observe a significantly slower electro-optical FK response extended to more than $10 \mathrm{ps}$ which is controlled by external field collapse, internal field screening, and suppressed recombination in an electric field. The measurements at different wavelengths provide an elegant tool for the discrimination between state filling effects and electroabsorption as well as for studying the time evolution (drift, trapping, and recombination) of the photogenerated space charge.

The authors thank C. B. Sørensen from III-V Nanolab for the samples. U.D.K. acknowledges support from the Danish Research Academy through a DANVIS grant and S.U.D. acknowledges support from the Deutsche Forchungsgemeinschaft. The work was also supported by the Danish Ministries of Industry and Research in the framework of CNAST.

${ }^{1}$ F. W. Smith, A. R. Calawa, C. L. Chen, M. J. Manfra, and L. J. Mahoney, IEEE Electron Device Lett. 9, 77 (1988).

${ }^{2}$ F. W. Smith, H. Q. Lee, V. Diadiuk, M. A. Hollis, A. R. Calawa, S. Gupta, M. Frankel, D. R. Dykaar, G. A. Mourou, and T. Y. Hsiang, Appl. Phys. Lett. 54, 890 (1989).

${ }^{3}$ S. Gupta, M. Y. Frankel, J. A. Valdmanis, J. F. Whitaker, G. A. Mourou, F. W. Smith, and A. R. Calawa, Appl. Phys. Lett. 59, 3276 (1991).

${ }^{4}$ C. Moglestue, J. Rosenzweig, J. Kuhl, M. Klingenstein, M. Lambsdorff, A. Axmann, Jo. Schneider, and A. Hülsmann, J. Appl. Phys. 70, 2435 (1991).

${ }^{5}$ S. Y. Chou and M. Y. Liu, IEEE J. Quantum Electron. 28, 2358 (1992).

${ }^{6}$ J. K. Luo, H. Thomas, D. V. Morgan, and D. Westwood, J. Appl. Phys. 79, 3622 (1996).

${ }^{7}$ M. O. Manasreh, D. C. Look, K. R. Evans, and C. E. Stutz, Phys. Rev. B 41, 10272 (1990).

${ }^{8}$ B. R. Bennett, R. A. Soref, and J. A. del Alamo, IEEE J. Quantum Electron. QE-26, 113 (1990).

${ }^{9}$ U. Siegner, R. Fluck, G. Zhang, and U. Keller, Appl. Phys. Lett. 69, 2566 (1996).

${ }^{10}$ M. Ruff, D. Streb, S. U. Dankowski, S. Tautz, P. Kiesel, B. Knüpfer, M. Kneissl, N. Linder, G. H. Döhler, and U. D. Keil, Appl. Phys. Lett. 68, 2968 (1996).

${ }^{11}$ W. Sha, J.-K. Rhee, T. B. Norris, and W. J. Schaff, IEEE J. Quantum Electron. 28, 2445 (1992).

${ }^{12}$ U. D. Keil and D. R. Dykaar, IEEE J. Quantum Electron. QE-32, 1664 (1996). 